\title{
GRAVITATIONAL LENSING BY STARS AND MACHOS AND THE ORBITAL MOTION OF THE EARTH
}

\author{
M. Hosokawa ${ }^{1}$, K. Ohnishi ${ }^{2}$, T. Fukushima ${ }^{3}$ and M. Takeutit ${ }^{4}$, \\ ${ }^{1}$ Communications Research Laboratory, \\ Koganei, Tokyo 184, Japan. e-mail hosokawa@crl.go.jp \\ ${ }^{2}$ Kansai Advanced Research Center, Communications Research Laboratory, \\ Kobe 651-24, Japan. e-mail ohnishi@crl.go.jp \\ ${ }^{3}$ National Astronomical Observatory, \\ Mitaka,Tokyo 181, Japan. e-mail toshio@spacetime.mtk.nao.ac.jp \\ ${ }^{4}$ Astronomical Institute, Tohoku University, \\ Aoba-ku,Sendai,980 Japan.e-mail i4aOs4d@JPNTOHOK.ecip.tohok.ac.jp
}

\begin{abstract}
We showed that it is feasible to measure the mass of a single star by observing the variation of gravitational deflection caused by the orbital motion of the Earth. When the distance of a star is less than $60 \mathrm{pc}$ and some appropriate sources are within $1 \mathrm{arcsec}$. in its background, not only the distance but also the mass of the star may be determined by measuring the deflection with an accuracy of $10 \mu$ arcsec. In the case of photometric microlensing by a MACHO, the observation of astrometric gravitational deflection is also useful. By measuring the separation between the primary image and the secondary image, the ratio of mass to distance of the MACHO will be obtained. Further, the orbital motion of the Earth modifying the light curve of the source is discussed.
\end{abstract}

\section{Introduction}

Measurement of a stellar mass, especially that of a single star, is very difficult. In principle, gravitational light deflection by a star depends on its mass. Therefore this effect can be used to measure the mass of the star. Unfortunately, the deflection angle itself cannot be measured unless the configuration of the source, deflector and observer changes. That makes it difficult to apply this effect.

On the other hand, much attention has been paid to another aspect of the gravitational lensing, that is called the microlensing. The possibility of microlensing by galactic dark halo objects was discussed by Paczynski and Griest (Paczynski,1987; Griest 1991). Recently, many candidate events of this phenomena caused by MACHOs are found (Alcock et al. 1993; Aubourg et al. 1993; Udalski et al. 1993). In spite of these successful observations, this method has some limitations. The alignment must be so well that the event rate of this photometric phenomenon is very small, the order of a millionth per year per star. For the determination of the mass of the lensing matter, some assumptions are needed. Also it is impossible to know which star will be lensed.

Now, the direct observation of the variation of the gravitational deflection is becoming feasible thanks to the development of high accuracy astrometric observation methods. In the near future, an accuracy of $10 \mu$ as is expected to be achieved by some VLBI and optical interferometer (Lestrade et al. 1992; Sasao et al. 1993; Shao and Colavita 1992). The theory and an application of this effect is discussed in Section 2 (Hosokawa et al. 1993). The gravitational deflection will also be detectable in the case of microlensing phenomenon. This issue is discussed in section 3.

\section{Parallactic Variation of Gravitational Deflection}




\subsection{THEORY}

Consider that a light emitted from a point source $S$ was deflected by the gravitational field of a foreground star $P$ and reached to the Observer $\mathrm{O}$. The distance between $\mathrm{O}$ and $P$, and that between $\mathrm{O}$ and $S$ is denoted by $D$ and $D s$, respectively. Here we consider the case that the separation angle between $\mathrm{P}$ and $S$ is about the order of one arcsecond, and the source $S$ is sufficiently far from the deflector P. Configuration of the S, P and $\mathrm{O}$ is shown in Fig.1. The angle $\beta$ is the true separation between $\mathrm{P}$ and $S$ in the absence of gravitational lensing (the angular separation in the source plane) while $\theta$ is the apparent separation (that in the lens plane). The relation between $\beta$ and $\theta$ is expressed as

$$
\theta=\beta+\frac{4 G M}{c^{2} D \theta},
$$

where $G$ is the gravitational constant, $c$ is the speed of light and $M$ is the mass of the deflector $P$ (Schneider et al. 1992).

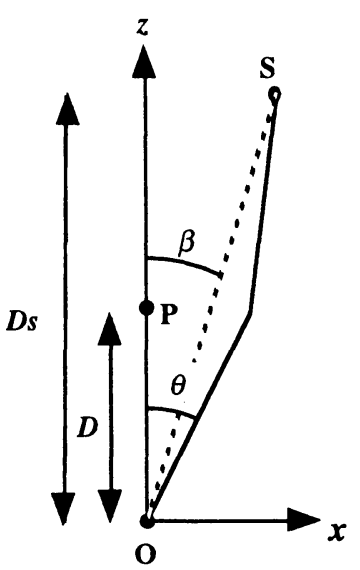

Fig. 1. Configuration of the gravitational lensing

Next, we consider the case that the observer shifts its position by $\boldsymbol{r}(\boldsymbol{x}, \boldsymbol{y}, \boldsymbol{z})$. According to this shift, the position of the source relative to the deflector will change. Let us denote the change of the source's position in the source plane by $\Delta \beta$ and that in the lens plane by $\Delta \theta$, respectively. In the case $|r| \ll D \theta$, these quantities are obtained as follows by expanding the deflection angle in the first order.

$$
\Delta \theta x=\Delta \beta x\left(1-\frac{4 G M}{c^{2} D \theta^{2}}\right), \quad \Delta \theta y=\Delta \beta y\left(1+\frac{4 G M}{c^{2} D \theta^{2}}\right) .
$$

Here, the position shift along the line of sight $z$ will make no change in the angular position in this order. Note that the angular shift along the source direction is suppressed by the change of the impact parameter while that across the source direction is enhanced by $\beta / \theta$ owing to the deflection itself. So they are not parallel in general. Using this effect, we can determine the mass and the distance of the deflector simultaneously. If we take the mass of the deflector $M$ as 10 $M_{\text {sun }}, \theta$ as 1 arcsecond, $D$ as $100 \mathrm{pc}$ and $|r|$ as the order of one $A U$, then $\Delta \beta_{\mathbf{x}} \sim \Delta \beta_{\mathbf{y}} \sim 10$ mas and $4 G M / c^{2} D \theta^{2}$ becomes the order of $1 / 1000$. Hence, for a star with $D \sim 100 \mathrm{pc}$ and $M \sim 10$ $M_{\text {sun }}$, this effect will amount to $10 \mu$ as, the same value as the expected accuracy mentioned before. By solving Eqs (2), we obtain the following equations.

$$
M=\frac{c^{2} \theta^{2}}{8 G}\left(\frac{x}{\Delta \theta x}-\frac{y}{\Delta \theta y}\right), \quad D=\frac{1}{2}\left(\frac{x}{\Delta \theta x}+\frac{y}{\Delta \theta y}\right) .
$$

Then, we will discuss on two applications of these formulae to determine the mass of a star.

In the case that a pair of sources can be found close enough to the deflector, the change of the separation angle between two sources caused by the position shift of the observer is proportional to the mass of the deflector. Therefore in this case, we need not decompose the angular shift of the separation into two components. Let two sources $S_{1}$ and $S_{2}$ be close to a deflector $P$. 


\subsection{VARIATION DUE TO ANNUAL PARALLAX}

In order to detect this variation of gravitational deflection, it can be considered to make use of the orbital motion of the Earth fqr the position shift of the observer. In this case, the parallactic ellipse of the deflector will suffer a shear from the gravitational deflection. According to the convention used in the present section, we choose the position of the deflector as a origin and express the parallactic ellipse as the trajectory of the motion of the source relative to the deflector. The case that the deflector $P$ and the source $S$ is in the same ecliptic latitude is illustrated in Fig. 3, where $\Pi$ is the annual parallax of the deflector and $\varepsilon=4 G M / c^{2} D \theta^{2}$. Hence, if we assume an accuracy to be better than $10 \mu$ as, for a stars of $M>10$

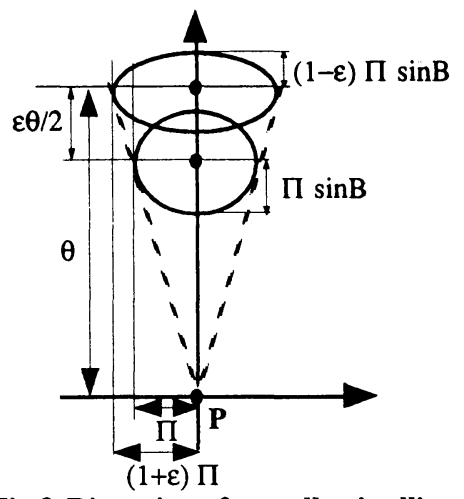

Fig.3. Distortion of parallactic ellipse $M_{\text {sun }}$ and $D<100 \mathrm{pc}$, or $M>5 M_{\text {sun }}$ and $D<60 \mathrm{pc}$, and that we have some appropriate sources within 1 arc second in its background, its distance and mass will be determined by detecting the shear of the parallactic ellipse caused by the gravitational deflection. This may be the most practical application of this effect.

\section{Microlensing and Gravitational Deflection}

In the case of microlensing by the MACHOs, there are four unknown parameters. These are the mass $M$ and the distance $D$ of the MACHO, the relative proper motion between the background source and the MACHO $\mu$, and the smallest impact parameter $r_{m}$. The source is assumed to be sufficiently far from the lensing matter, as in sec. 2 . These quantities can be rewritten as the set $D, \Theta_{0}, A_{\max }$ and $t_{e}$, where $\Theta_{0}$ is the angular radius of Einstein Ring, $\mathrm{A}_{\max }$ is the largest amplification of the luminosity and $t_{e}$ is the characteristic time scale of the event. By observing the light curve of the microlensing event, however, we can obtain only two parameters; $A_{\max }$ and $t_{e}$. In order to obtain the rest of these parameters, the observation of the gravitational deflection will play an important role.

In the microlensing, the formulae on the luminosity amplifications and the deflection angles of the primary image and the secondary image of the source are well known (Paczynski,1987). As an example, the trajectories of the primary image and the secondary image in the case that the least impact parameter $r_{m}$ is $0.2 D \Theta_{0}$ is illustrated in Fig.4 (Hosokawa, et al. 1994; Hog, et al. 1994). Note that, if we take $M$ as $0.1 \mathrm{M}_{\text {sun }}$ and $D$ as 10 $\mathrm{kpc}$, we obtain the value of $\Theta_{0}$ as 0.3 mas. Then, the separation between the primary image and the secondary image of the source will be larger than 0.6 mas even at the epoch of the closest approach. Though the luminosity of the source is very faint because of its distance, such as 20 mag. in the case of the stars in Large Magellanic Cloud, this angle itself is separable enough in the resolution of recent optical interferometers. As for the luminosity enhancement, that of the secondary image is amplified twice of the original one in the epoch of the closest approach (Fig.5). Though this amplification will

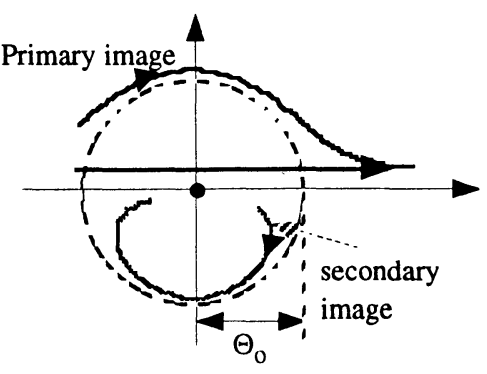

Fig.4. Trajectories of primary image and secondary image in microlensing 
make it easier to observe these two images, it may be difficult for the interferometers of today and near future to observe a binary of the $19 \mathrm{mag}$. with the resolution of 0.1 mas. However, the observation facilities of the interferometer is being advanced rapidly (Shao, M., Colavita, M.M., 1992). If a powerful observation facility that enable us to resolve these images are completed, and the real time analysis of the photometric microlensing search, that could tell us which stars the candidates are, is realized in the future, then the separation between the primary image and the

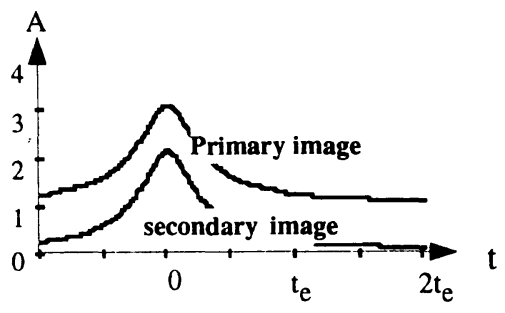

Fig.5. Light curves of primary image and secondary image in microlensing secondary image of the source may be measured. To resolve these two images nas been considered by Gould (1992), who proposed that this high resolution observation could be applied to confirm the microlensing events caused by the heavy MACHOs $\left(10^{3} \mathrm{M}_{\text {sun }}>\mathrm{M}>10^{3} \mathrm{M}_{\text {sun }}\right)$. Now, many candidate events have been observed and the masses of the MACHOs are thought to be, under some assumptions, much lighter than in that range. So we should seek for the way to apply this high resolution observation to the MACHOs in this light mass range.

Further, it should be noted that the orbital motion of the Earth modifies the light curve of the source. In this modification, the acceleration of the Earth is more useful than the variation of its position. In a typical duration of the events, about 30 days, the variation of the Earth's velocity amounts to $15 \mathrm{~km} / \mathrm{s}$. This effect causes not only the seasonal variation of event rate (Griest 1991) but also the asymmetry of the light curve. In the case that the acceleration of the Earth is almost parallel or anti-parallel to the proper motion of the MACHO relative to the source and that the event duration is short enough to regard the acceleration as almost constant during that period, this modification becomes maximum and is easily obtained. For example, the epoch of maximum brightness shifts 12 hours maximally from the center of profile when the event duration is 30 days and the transverse velocity of MACHO is $100 \mathrm{~km} / \mathrm{sec}$.

In conclusion, to detect the variation of gravitational light deflection is within reach. This can be used for the mass measurement of the stars and MACHOs. In the latter, to resolve the primary image and the secondary image is needed. Therefore it might be more difficult than the measuring the position shift as in the former. In both cases, the orbital motion of the Earth will be useful for making detectable variations in observable quantities.

\section{References}

Gould, A.,1992, Apj, 392, 442.

Griest, K., 1991, ApJ, 366, 413.

Høg, E., Novikov,I.D., and Polnarev,A.G., 1994, A\&A, to be published.

Hosokawa, M., Ohnishi, K., Fukushima, T. and Takeuti, M. , 1993, A\&A 278, L27.

Hosokawa, M., Ohnishi, K., Fukushima, T. and Takeuti, M. , 1994, Proceedings of the Third

Workshop on General Relativity and Gravitation, Tokyo, p.307

Lestrade, J., Phillips, R.B., Preston,R.A., Gabuzda, D.C., 1992,A\&A 258,112.

Paczynski, B., 1987, ApJ, 304, 1.

Sasao, T., Kawano.N.,Hara.T., et al., 1993, Proceedings of iRiS '93, CRL, TOKYO, p.91.

Schneider, P., Ehlers, J., Falco, E.E., 1992, Gravitational Lenses, Springer - Verlag.

Shao, M., Colavita, M.M., 1992, A\&A 262, 353.

Udalski, A., et al., 1993, ActaAstr. 43, 289. 